Check for updates

Cite this: RSC Adv., 2018, 8, 32538

\title{
A novel surface plasmon resonance sensor based on a functionalized graphene oxide/molecular- imprinted polymer composite for chiral recognition of L-tryptophan
}

\begin{abstract}
Xiaoyan Xu, (D) a Yi Zhang, ${ }^{a}$ Bingfeng Wang, ${ }^{b}$ Lin Luo, ${ }^{a}$ Zhenlin $\mathrm{Xu}^{\mathrm{a}}$ and Xingguo Tian ${ }^{\star a}$
Herein, a novel surface plasmon resonance (SPR) sensor based on a functionalized graphene oxide (GO)/ molecular-imprinted polymer composite was developed for the chiral recognition of L-tryptophan ( $L$ Trp). The composite's recognition element was prepared via a facile and green synthesis approach using polydopamine as both a reducer of $\mathrm{GO}$ and a functional monomer as well as a cross-linker for molecular imprinting. The composite was characterized via Fourier transform infrared spectroscopy, scanning electron microscopy, X-ray diffraction, and Raman spectroscopy. After attaching the composite onto the gold surface of an SPR chip, the sensor was characterized using contact-angle measurements. The sensor exhibited excellent selectivity and chiral recognition for the template (i.e., L-Trp). Density functional theory computations showed that the difference in hydrogen bonding between the

composite element and L-Trp and D-Trp played an important role in chiral recognition.
\end{abstract}

Received 25th July 2018

Accepted 12th September 2018

DOI: $10.1039 / c 8 r a 06295 a$

rsc.li/rsc-advances

\section{Introduction}

Chiral recognition remains vitally important in the medical science, biochemistry, and pharmaceutics fields because of the dissimilar or opposite performances exhibited by enantiomers of chiral molecules in biological systems. ${ }^{1} \mathrm{~L}$-Tryptophan (L-Trp) is an essential amino acid and precursor of the neurotransmitter serotonin in humans and animals, and its imbalance or deficiency can cause several chronic diseases. ${ }^{2,3}$ Hence, a practical, rapid and available method for the chiral recognition of Trp enantiomers is important to ensure their safe and effective use. Various methods for the chiral recognition of Trp enantiomers have been reported, including high-performance liquid chromatography (HPLC), electrochemical detection, colorimetric analysis, and fluorescence detection. ${ }^{4-7}$ However, the disadvantages of these approaches, which include expensive instrumentation, laborious sample pretreatments, and complicated derivatization procedures, impede their broad application. ${ }^{2,8}$

As powerful analytical tools, surface plasmon resonance (SPR) sensors have enjoyed widespread attention over the past two decades because of their high sensitivity, low cost, and compact design. ${ }^{9}$ SPR, a refractive index-based detection technique for real-time and label-free monitoring of ligand-receptor

${ }^{a}$ Guangdong Provincial Key Laboratory of Food Quality and Safety, College of Food Science, South China Agricultural University, Guangzhou 510642, PR China. E-mail: xingguot@scau.edu.cn

${ }^{b}$ Department of Applied Chemistry, College of Materials and Energy, South China Agricultural University, Guangzhou 510642, PR China interactions on metal surfaces, ${ }^{10-13}$ has been extended to the molecular imprinting field. ${ }^{14}$ Molecular imprinting is an artificial molecular recognition technique based on the "molecular lock and key" principle. ${ }^{15}$ This technique involves polymerization around the target molecule, leading to the formation of specific cavities in the cross-linked polymer matrices. Molecular-imprinted polymers (MIPs) are good elements for recognition and detection in SPR sensors because of their substantial advantages, including desirable selectivity, mechanical/chemical stability, and low cost, over other detection elements. In recent years, MIPs have been extensively reported as surface modification materials for chips in the fabrication of different kinds of SPR sensors. ${ }^{16-18}$ However, in the analysis of small molecules, MIP-decorated sensor chips suffer from insufficient sensitivity because small molecules can only induce small changes in the refractive index. Thus, the development of simple and versatile strategies for amplifying SPR sensing has been proven as a challenging task. ${ }^{19}$

Graphene (Gr), a two-dimensional $\mathrm{sp}^{2}$-hybridized carbon material comprising a single sheet of hexagonally packed carbon atoms, has a number of advantages, including large specific surface area, good electrical conductivity, and superlative mechanical strength, making $\mathrm{Gr}$ a promising candidate for enhancing SPR signals. ${ }^{20,21}$ These special characteristics impart Gr/MIP composites with higher affinity and sensitivity toward the target analytes and provide a more homogeneous distribution of recognition sites. ${ }^{22}$ Despite the strong application potential of Gr/MIP composites, Gr tends to irreversibly agglomerate via van der Waals forces during the drying 
process. ${ }^{23}$ Accordingly, effective and mild methods for functionalizing $\mathrm{Gr}$ to improve its stability and dispersity are required. ${ }^{24}$

Dopamine, commonly known as a hormone and neurotransmitter, has recently aroused considerable interest because it is also a unique molecule that mimics mussel adhesive proteins. ${ }^{25}$ Inspired by the high content of 3,4-dihydroxy-Lphenylalanine and lysine found in mussel adhesive proteins, which is predominantly located at the plaque-substrate interface, researchers have developed a material-independent surface functionalization strategy involving the selfpolymerization of dopamine to form chemically active adherent films on virtually all types of inorganic and organic surfaces. $^{26,27}$ The adherent polydopamine (PDA) coating can react with various functional groups, including thiols and amines, via Michael addition or Schiff base reaction. ${ }^{28,29}$ Because of the aforementioned breakthrough, PDA can not only be used as a natural barrier against the interaction of negatively charged particles to prevent the aggregation of Gr but also serve as a functional monomer and cross-linker for molecular imprinting.

In our previous study, ${ }^{30}$ we combined the merits of the molecular imprinting technology and SPR sensing to detect Sudan dyes sensitively. Herein, we extend this sensing platform to develop amplified SPR sensors based on functionalized $\mathrm{Gr}$ / MIP composites for the chiral recognition of Trp enantiomers. To the best of our knowledge, this research is the first attempt toward employing PDA as a functional monomer, cross-linker, and reducer of graphite oxide (GO) simultaneously. The fabrication scheme of the Gr/MIPSPR sensor is illustrated in Scheme 1. First, the composite was synthesized via the selfpolymerization of dopamine using a one-pot approach in the presence of template L-Trp. Then, various characterization techniques, including Fourier transform infrared (FTIR) spectroscopy, scanning electron microscopy (SEM), X-ray diffraction (XRD), and Raman spectroscopy were used to verify the successful synthesis of the composite. After characterization,

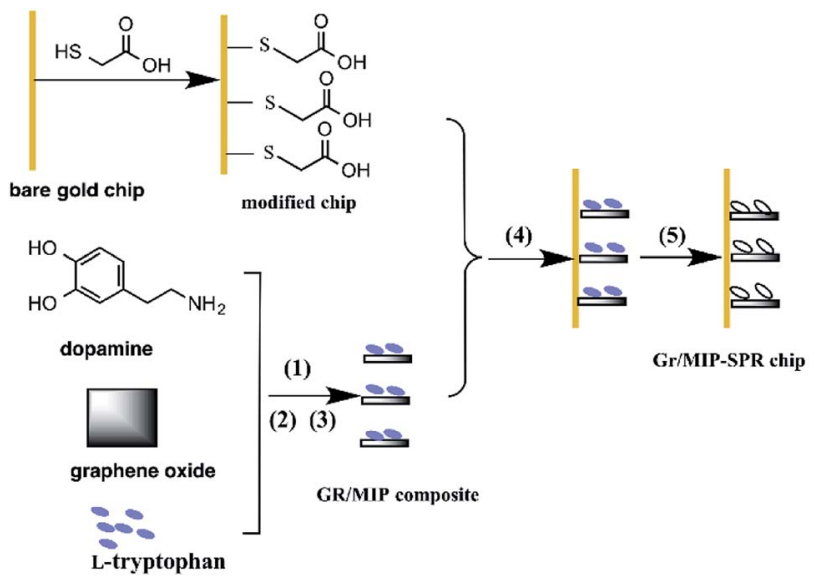

(1) self-polymerization of dopamine; (2)reduction of graphene oxide; (3) imprinting of L-tryptophan;(4) in situ polymerization;(5) remove template

Scheme 1 Schematic representation of preparation of Gr/MIPSPR sensor chip via one-pot in situ polymerization. the Gr/MIP composite was immobilized onto the gold surface of an SPR chip as a molecular recognition element for the enantioseparation and recognition of $\mathrm{L}_{\text {-Trp }}$ and D-Trp. Kinetic analysis was subsequently performed to examine the interaction kinetics between the Gr/MIPSPR sensor and the Trp enantiomers. Additionally, the selectivity, reusability, and recognition mechanism of the Gr/MIPSPR sensor were investigated.

\section{Experimental}

\section{Chemicals and materials}

Graphite was purchased from the Damao Chemical Reagent Factory (Tianjin, China). Concentrated sulfuric acid $\left(\mathrm{H}_{2} \mathrm{SO}_{4}\right)$ and $30 \%$ hydrogen peroxide $\left(\mathrm{H}_{2} \mathrm{O}_{2}\right)$ were purchased from the Guangzhou Chemical Reagent Factory (Guangzhou, China). LTrp, D-Trp, dopamine hydrochloride, tris(hydroxymethyl)aminomethane (Tris), ammonium persulfate (APS), and $\beta$-mercaptoethylamine were obtained from Macklin Biochemical Co., Ltd. (Shanghai, China). All chemicals were of analytical grade and were used directly without further treatment. Milli-Q ultrapure water (resistivity $=18.2 \mathrm{M} \Omega \mathrm{cm}$ ) was used throughout the experiments. The gold-coated glass SPR sensor chips (Au thickness of $\sim 50 \mathrm{~nm}$ ) were obtained from Reichert Inc. (NY, USA).

\section{Instrumentation}

The SPR measurements were performed on a Biacore3000 SPR system (Biacore, Uppsala, Sweden). SEM imaging was performed using an XL30 TMP environmental scanning electron microscope (Philips-FEI, the Netherlands). The FTIR spectra (4000-500 $\mathrm{cm}^{-1}$ ) were recorded using a Vertex 70 spectrometer (Bruker, Germany) with a resolution of $0.4 \mathrm{~cm}^{-1}$. Samples for FTIR analysis were prepared as $\mathrm{KBr}$ pellets. Transmission electron microscopy (TEM) images were acquired on a JEM2100F transmission electron microscope (JEOL, USA). The Raman spectra were recorded by a LabRam HR 800 (Horiba, France). The contact angle of the MIPSPR sensor was determined using a JC-2000C1 tensiometer (Shanghai Zhongchen, China). The water used to prepare buffer and other aqueous solutions was purified using a Milli-Q system (Millipore, USA).

\section{Surface modification of the SPR chip}

Prior to modification, the bare gold surface of the SPR chip was first cleaned using $10 \mathrm{~mL}$ of fresh piranha solution $\left[\mathrm{H}_{2} \mathrm{SO}_{4}\right.$ $(98 \%): \mathrm{H}_{2} \mathrm{O}_{2}(30 \%)=3: 1 \mathrm{v} / \mathrm{v}$ ] for $5 \mathrm{~min}$, followed by thorough rinsing with copious amounts of deionized water and ethanol. The chip was subsequently placed into $20 \mathrm{~mL}$ of a $10 \mathrm{mM}$ solution of $\beta$-mercaptoethylamine in ethanol, allowed to form a self-assembled monolayer (SAM) for $24 \mathrm{~h}$, rinsed with ethanol, and dried under a nitrogen atmosphere. The modified SAM chip was immediately used for polymer preparation.

\section{One-pot in situ preparation of Gr/MIP composites coated onto the SPR chip}

After being synthesized according to the method of Hummers and Marcano, ${ }^{31} \mathrm{GO}$ (6 mg) was added to $20 \mathrm{~mL}$ of $10 \mathrm{mM}$ Tris 
(pH 8.5) buffer solution and sonicated in an ice-water bath for $30 \mathrm{~min}$ to ensure uniform dispersal. Next, $10 \mathrm{mg}$ of L-Trp (template), $7.6 \mathrm{mg}$ of DA, and $20 \mathrm{mg}$ of APS (initiator) were added to the GO suspension. The sample was then mechanically shaken and sonicated for 5 min to obtain a homogeneous polymerization mixture. The modified SPR chip was transferred to the polymerization mixture and baked in a vacuum oven at $60{ }^{\circ} \mathrm{C}$ for $12 \mathrm{~h}$. Finally, the composite-coated SPR chip was washed using $0.1 \mathrm{M} \mathrm{HCl} /$ methanol $(1: 1 \mathrm{v} / \mathrm{v})$ for $48 \mathrm{~h}$ with a Soxhlet extractor until no template was detected. For comparison, Gr/non-imprinted polymer (Gr/NIP) composites were also prepared using the same procedure but without adding a template.

\section{SPR measurements}

The Gr/MIP composites were immobilized via an aminecoupling reaction on a sensor chip (Biacore, Piscataway, NJ, USA) inserted into the flow chamber of a Biacore 3000 SPR system (Biacore, Uppsala, Sweden). SPR measurements were conducted at $25{ }^{\circ} \mathrm{C}$ at a flow rate of $20 \mu \mathrm{L} \mathrm{min}{ }^{-1}$ using phosphate-buffered saline with Tween (PBST; pH 8.0) as the running buffer. The data [expressed in response units (RU), which are directly related to the shift in the SPR angle] were acquired using the Biacore version 3.2 evaluation software package. A $0.1 \mathrm{M} \mathrm{HCl}$ solution was used for the regeneration step after the analytes were adsorbed onto the Gr/MIP-coated gold chip. The analysis sequence included four processes, and the time of each was controlled as follows: baseline (300 s), association (200 s), dissociation (100 s), and regeneration (60 s). Before the analysis procedure, PBST ( $\mathrm{pH}$ 8.0) was used to stabilize the surface of the gold chip until a stable base line was obtained.

\section{Computational simulations}

All theoretical computations in this research were performed using the density functional theory (DFT) method at the B3LYP/ 6-31G*level, as implemented in the Gaussian 09 software package running under a Linux operating system. The conformations of each isolated compound (L-Trp, D-Trp, and PDA) and their complexes in the prepolymerization process were optimized. The interaction energies $\Delta E$ were obtained using the following equation:

$$
\Delta E=E_{\text {complex }}-E_{\mathrm{Trp}}-E_{\mathrm{PDA}}
$$

where $E_{\text {complex }}$ is the total energy of the simulated Trp-PDA complex, $E_{\operatorname{Trp}}$ is the minimum energy of L-Trp or D-Trp, and $E_{\mathrm{PDA}}$ is the minimum energy of PDA.

\section{Results and discussion}

\section{Characterization}

Fig. 1A shows the FTIR spectra of the Gr/MIP and Gr/NIP composites. The spectra of the Gr/MIP and Gr/NIP composites were approximately the same, indicating that the $\mathrm{L}_{\mathrm{L}}$ Trp template was completely removed from the Gr/MIP composite and that the two composites exhibited similar chemical constitutions.

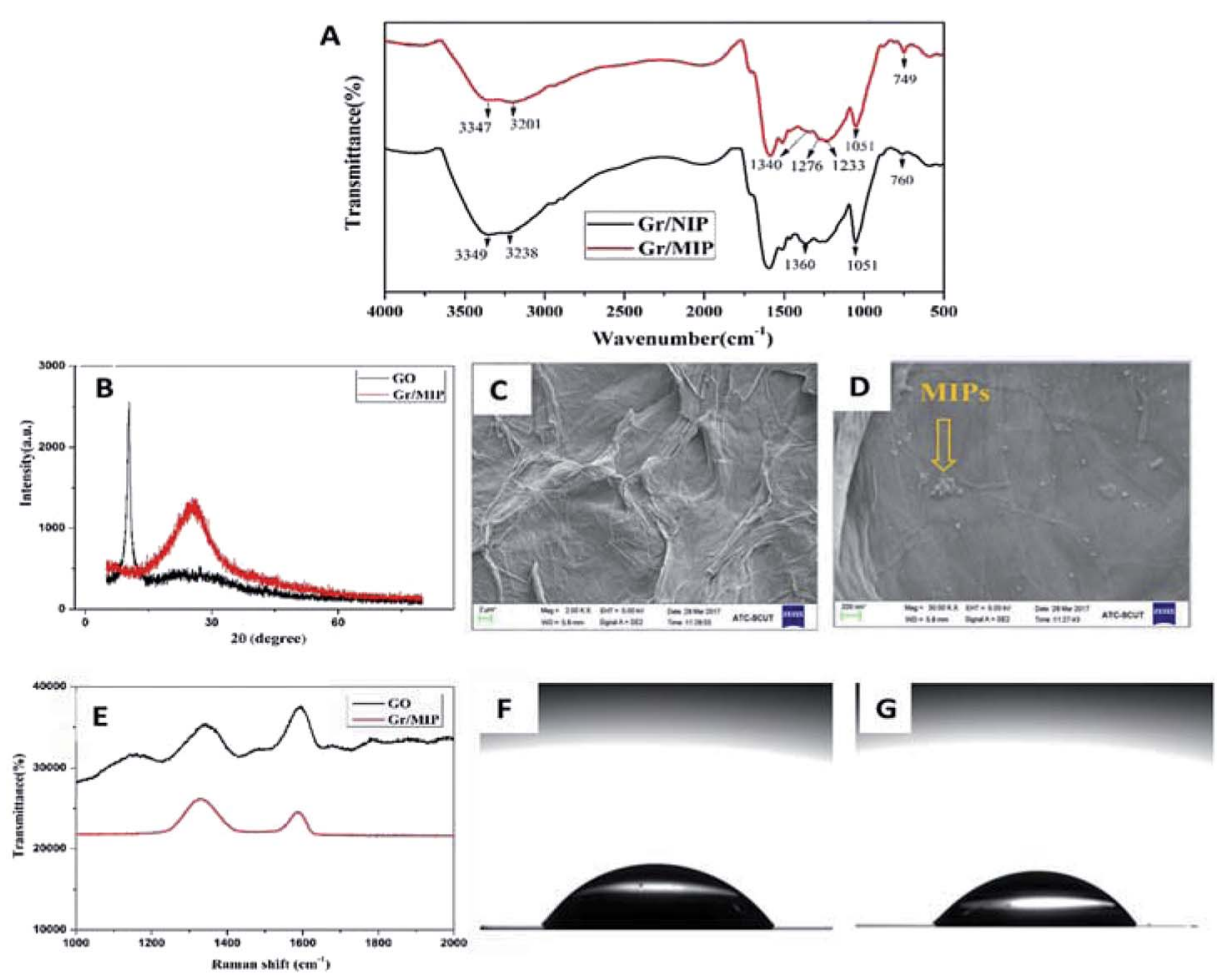

Fig. 1 (A) FTIR spectra of Gr/MIP and Gr/NIP, (B) XRD patterns of GO and Gr/MIP, SEM images of (C) GO and (D) Gr/MIP, (E) Raman spectrum of $\mathrm{GO}$ and Gr/MIP, contact angle measurements of (F) bare and (G) Gr/MIP-coated SPR chip. 
The two adsorption peaks in the range $3200-3400 \mathrm{~cm}^{-1}$ of both spectra, which correspond to $\mathrm{N}-\mathrm{H}$ stretching vibrations, confirm that PDA was involved in the polymerization of the molecular imprinting. In addition, minor decrease in the intensity and red shift of the two peaks (from $3349 \mathrm{~cm}^{-1}$ to $3347 \mathrm{~cm}^{-1}$ and from $3238 \mathrm{~cm}^{-1}$ to $3201 \mathrm{~cm}^{-1}$, respectively) were observed, which may be ascribed to the hydrogen bond interactions between PDA and template molecules.

Fig. 1B shows the XRD patterns of GO and the Gr/MIP composite. In the XRD pattern of GO, a characteristic peak appeared at $2 \theta=10^{\circ}$, revealing that oxygen functional groups had been introduced onto the graphite sheets and that the formation of GO from graphite powder was successful. ${ }^{32,33}$ After polymerization, this characteristic graphite peak disappeared and was replaced by a well-defined peak at $2 \theta=25^{\circ}$, which is ascribed to the reduction of GO to Gr via PDA modification. ${ }^{34}$

The morphology and microstructure of GO and the Gr/MIP composites were investigated. GO (Fig. 1C) presents a typical layer-like structure with some wrinkles. However, the SEM image of the Gr/MIP composites (Fig. 1D) revealed that the wrinkles disappeared and that the surface of the Gr sheets was covered with a spherical structure comprising MIP nanoparticles. These results clearly illustrated that GO was reduced by PDA and that the Gr/MIP composites were successfully synthesized.

The Raman spectra in Fig. 1E further confirmed the successful grafting of Gr/MIP composites onto the GO sheets. The Raman spectrum of GO exhibited two strong peaks at 1350 and $1590 \mathrm{~cm}^{-1}$ corresponding to the D- and G-bands, respectively. In general, the G-band is associated with the vibration of $\mathrm{sp}^{2}$ carbon atoms in the two-dimensional hexagonal lattice, whereas the intensity of the D-band indicates the degree of disorder or the number of structural defects in graphene oxide. ${ }^{35}$ In addition, the intensity ratio $\left(I_{\mathrm{D}} / I_{\mathrm{G}}\right)$ is commonly used to reflect the extent of graphitization of carbonaceous materials and their defect density. The $I_{\mathrm{D}} / I_{\mathrm{G}}$ ratios of GO and $\mathrm{Gr} / \mathrm{MIP}$ composites were 0.712 and 1.203 , respectively, indicating an increase in disorder. This change can be attributed to the formation of covalent bonds between grand PDA.

Water contact-angle measurements were performed to characterize the sensor after the Gr/MIP composite was attached to the chip surface. As shown in Fig. 1F and G, the contact angle of a water droplet on the chip surface decreased from $56.50^{\circ}$ to $44.25^{\circ}$ upon the introduction of the Gr/MIP composite. These results indicate that polymerization was accomplished on the SPR chip and resulted in an increase in the surface hydrophilicity.

\section{Effect of pH}

The $\mathrm{pH}$ of a medium strongly affects sensor-analyte affinity. The sensor responses to $2.5 \mathrm{mM}$ L-Trp in PBST buffer solutions with various $\mathrm{pH}$ values are shown in Fig. 2 . The best response signal was observed at $\mathrm{pH}$ 8.0, which indicates that the binding of L-Trp to the SPR chip surface progressively increased as the $\mathrm{pH}$ was increased to 8.0. Hence, $\mathrm{pH} 8.0$ was selected as the optimum $\mathrm{pH}$ value.

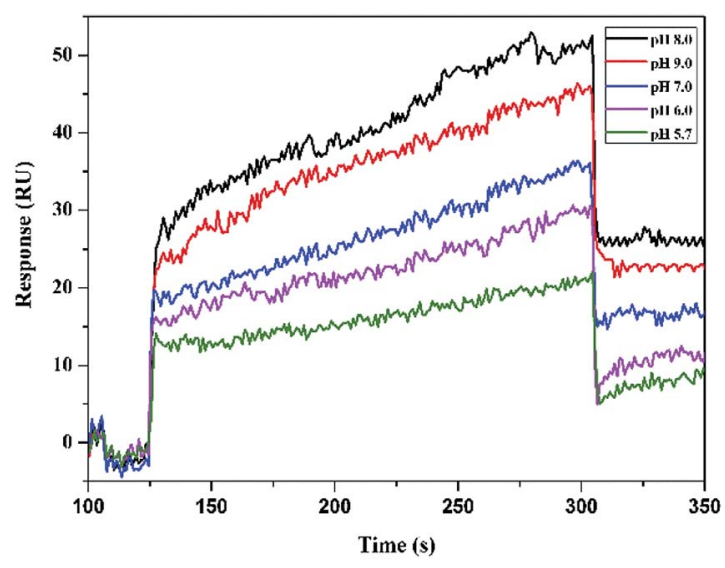

Fig. 2 Effect of pH on SPR signal (in the presence of $2.5 \mathrm{mmol}^{-1} \mathrm{~L}-\operatorname{Trp}$ ).

\section{Binding performance}

To test the chiral recognition of the SPR sensor for Trp enantiomers, we performed experiments for rebinding different concentrations of $\mathrm{L}, \mathrm{D}$-Trp to the sensor chip using the same procedures and the SPR sensor. The relations between the concentrations of L-Trp and D-Trp and the senor signal are shown in Fig. 3A and B, respectively. With the increasing concentrations of L-Trp and D-Trp, the SPR signal increased. The SPR signal of L-Trp was clearly much stronger than that of D-Trp at the same concentration. These results demonstrated that the enantioselective recognition of the resultant Gr/MIPSPR sensor toward L-Trp can be achieved through imprinting template molecules.

Fig. 3C shows the linear rang for the detection of L-Trp with Gr/MIPSPR sensor. The calibration graph depicts that correlation is high $\left(R^{2}=0.9939\right)$. The limit of detection (LOD) and limit of quantitation (LOQ) values of the Gr/MIPSPR sensor were determined as $0.105 \mathrm{mmol} \mathrm{L}^{-1}$ and 0.350 respectively, according to the linear part of calibration curve with the $3 S / b$ formula where $S$ is standard deviation of response and $b$ is the slope of calibration curve $\left(0.150-2.50 \mathrm{mmol} \mathrm{L}^{-1}\right)$.

It can be seen in Fig. 3D, the SPR signal is almost two-fold higher in the case of Gr/MIP sensor in comparison to the single MIP sensor at the same L-Trp concentration. The reasons could be attributed to two aspects: first, the Gr/MIP composites gave more recognition sites for L-Trp on account of the high surface area of the spherical structure. Second, the high molecular weight and the high refractive index of the Gr/MIP composites might play an important role in the amplification of SPR signal.

For a better comparison, binding kinetic analysis was performed to determine the host-guest interaction between Trp enantiomers and the Gr/MIPSPR sensors during SPR measurements. The association rate constant $\left(k_{\mathrm{a}}\right)$, dissociation rate constant $\left(k_{\mathrm{d}}\right)$, association constant $\left(K_{\mathrm{A}}=k_{\mathrm{a}} / k_{\mathrm{d}}\right)$, and dissociation constant $\left(K_{\mathrm{D}}=1 / K_{\mathrm{A}}\right)$ were calculated using a simple one-to-one Langmuir binding model. $K_{\mathrm{D}}$ is usually used to evaluate and rank order strengths of the intermolecular interactions, where a smaller value of $K_{\mathrm{D}}$ indicates a higher binding affinity. ${ }^{36}$ The results summarized in Table 1 indicated that the chiral 

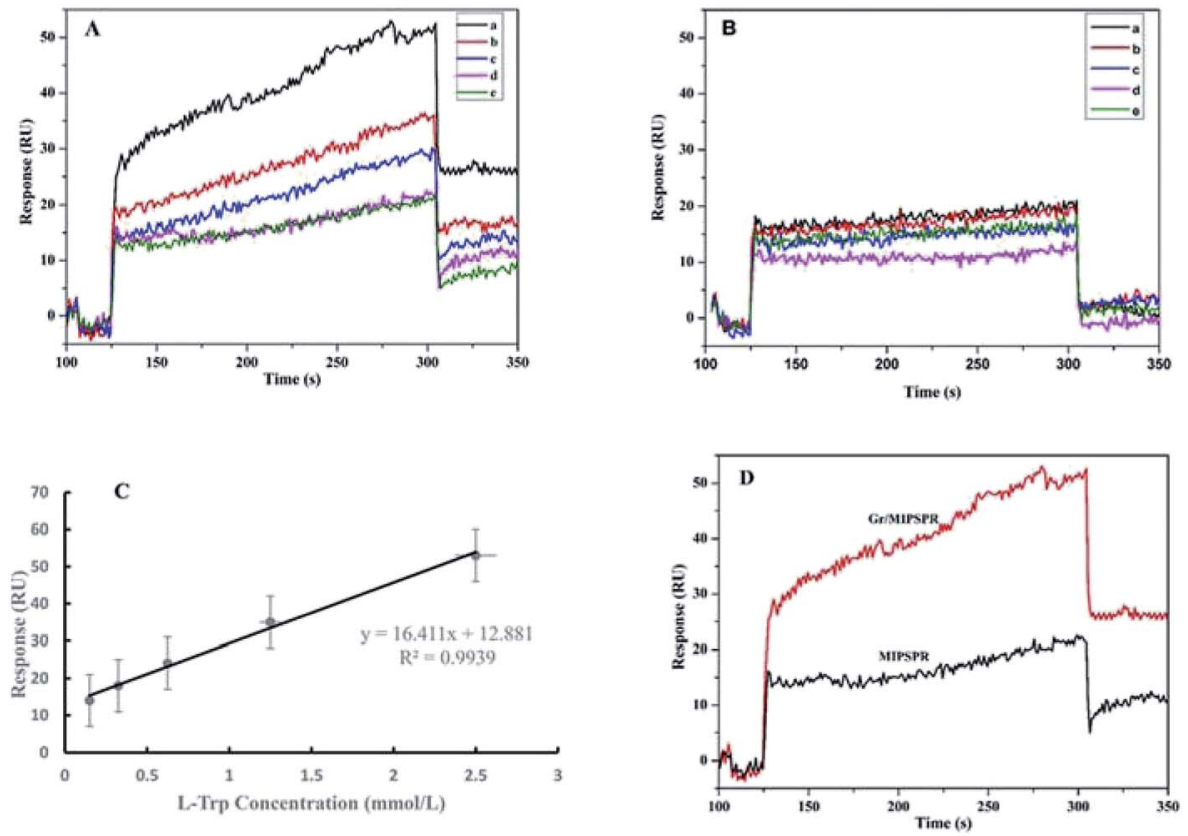

Fig. 3 SPR signals to different concentrations of (A) L-Trp and (B) D-Trp (a-e): 2.50, 1.25, 0.625, 0.325 and $0.150 \mathrm{mmol}^{-1}$; (C) the calibration curve for L-Trp on Gr/MIPSPR sensor; (D) SPR signals to $2.5 \mathrm{mmol} \mathrm{L}{ }^{-1}$ L-Trp on Gr/MIP-coated sensor and MIP-coated sensor. All SPR measurements were performed in phosphate-buffered saline with Tween (pH 8.0) at a flow rate of $20 \mu \mathrm{L} \mathrm{min}{ }^{-1}$.

recognition ability of the Gr/MIPSPR sensors toward L-Trp was substantially greater than that toward D-Trp.

\section{Recognition mechanism}

The elucidation of chiral recognition mechanisms is essential for chiral recognition and chiral separation. ${ }^{37}$ The recognition properties of the Gr/MIPSPR sensors are highly dependent on the quality of the binding sites contained in the matrix. In this study, DFT at the B3LYP/6-31G*(d,p) level was used to perform energy calculations for the final optimized conformations and evaluate the total $\Delta E$ value. The results are summarized in Table 2.

Hydrogen bonding is the dominant driving force for intermolecular interactions between the template and the functional monomer. The DFT approach was also used to investigate the

Table 1 Binding kinetic parameters

\begin{tabular}{lrlrll}
\hline Analyte & \multicolumn{1}{c}{$k_{\mathrm{a}}$} & \multicolumn{1}{c}{$k_{\mathrm{d}}$} & \multicolumn{1}{c}{$K_{\mathrm{A}}$} & \multicolumn{1}{c}{$K_{\mathrm{D}}$} & $R^{2}$ \\
\hline L-Trp & $7 \times 10^{6}$ & $9.4 \times 10^{-6}$ & $7 \times 10^{11}$ & $1.3 \times 10^{-12}$ & 0.99 \\
D-Trp & $2.1 \times 10^{5}$ & $0.8 \times 10^{-5}$ & $2.6 \times 10^{10}$ & $3.8 \times 10^{-11}$ & 0.58
\end{tabular}

Table 2 Interaction energy of Trp enantiomers with PDA

\begin{tabular}{lllll}
\hline & $E$ & & $\Delta E$ & \\
\cline { 2 - 3 } & a.u. & & a.u. & $\mathrm{kcal} \mathrm{mol}^{-1}$ \\
\hline D-Trp & -686.1691323 & - & - \\
L-Trp & -686.1681162 & & \\
PDA & -2052.8655456 & & -517.8672249 \\
D-Trp-PDA & -2739.85995182 & & -0.82527392 & - \\
L-Trp-PDA & -2739.86050274 & & -0.82684094 & -518.8505448
\end{tabular}

hydrogen bonding between Trp and PDA in implicit solvents (water); the results are shown in Fig. 4. We observed that the oxygen atoms of the carbonyl groups of the Trp enantiomer and the hydrogen atoms of the amino groups in the indole units of PDA could form two strong hydrogen bonds. The lengths of these two hydrogen bonds were 1.8 and $2.2 \AA$ for D-Trp and 1.8 and $2.0 \AA$ for L-Trp, respectively. In addition to the two hydrogen bonds formed by the carbonyl group as electron donors, the hydrogen atoms of the amino group in L-Trp accept an electron pair and interact with the oxygen atom of phenol hydroxyl via a hydrogen bond, the length of which was 1.8 A. Our experimental findings confirmed that the binding affinity between $\mathrm{L}^{-}$ Trp and PDA was stronger than that between D-Trp and PDA.

In this strategy, when DA was mixed with L-Trp and GO in a weak alkaline solution, GO was reduced to Gr for enhancing SPR signals due to the oxide self-polymerization of DA. Meanwhile, the template molecules can be trapped in the crosslinked polymeric network owing to the hydrogen bond interaction of L-Trp with DA. Chain branching and cross-linking in the PDA generated a three dimensional matrix toward the template molecules. ${ }^{38}$ After removal of the embedded template molecules, the created imprinted cavities in the polymer can
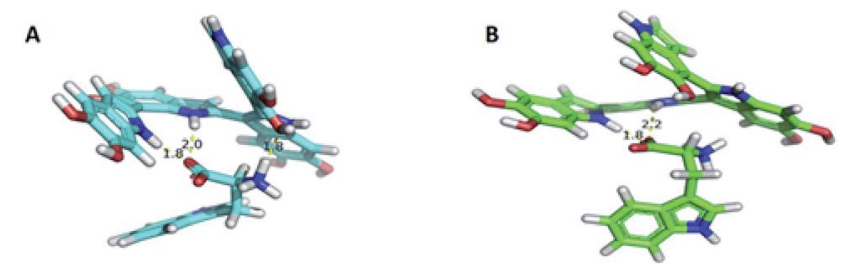

Fig. 4 Complex formed between (A) L-Trp and (B) D-Trp and polydopamine in implicit solvents (water). 


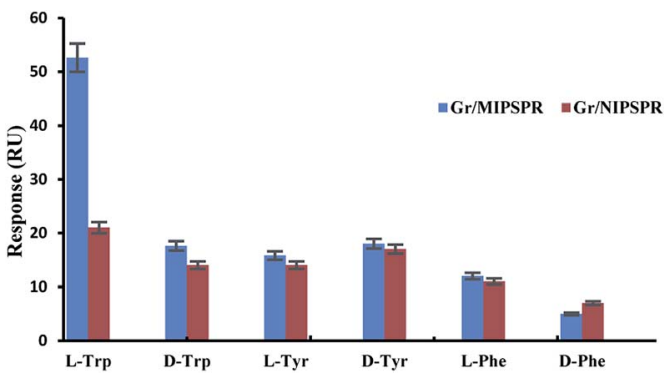

Fig. 5 Gr/MIPSPR and Gr/NIPSPR signals towards L- and D-Trp, L- and $D-T y r$, and L- and D-Phe (each of $2.5 \mathrm{mmol} \mathrm{L}^{-1}$ ).

recognize the template molecules based on shape selection and complementarity of the functional groups. ${ }^{39}$

\section{Selectivity}

The selectivity of the Gr/MIPSPR sensor was examined by comparing the SPR response toward Trp enantiomers with that of other chiral amino acids [tyrosine (Tyr) and phenylalanine (Phe)] in their mixture solution under the same conditions. As shown in Fig. 5, for the Gr/MIPSPR sensor, the signal values for L-Trp were significantly higher than those for D-Trp, L- and D-Tyr, and L- and D-Phe. In contrast, the NIPSPR sensor exhibited little selectivity for all analytes. Thus, the Gr/MIPSPR sensor chip had specific recognition sites that were complementary to template molecules in terms of the shape and positioning of functional groups. Although the Gr/NIPSPR sensor chip contains a sufficient number of functional groups, it lacks defined cavities to provide the selective binding of $\mathrm{L}$-Trp.

\section{Reusability}

The reusability of an SPR sensor is likely to be a key factor in improving process economics. ${ }^{40}$ To demonstrate the reproducibility of the Gr/MIPSPR sensor, we repeated three equilibration-adsorption-regeneration cycles with $2.5 \mathrm{mmol} \mathrm{L}^{-1} \mathrm{~L}-\mathrm{Trp}$ solution. As shown in Fig. 6, no substantial change in the SPR response was observed during the three cycles. Thus, the synthesized Gr/MIPSPR sensor can be reused at least three times with stable results.

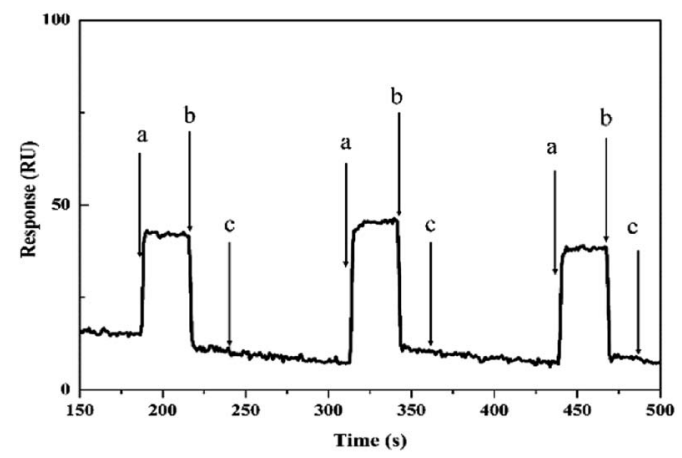

Fig. 6 Reusability of Gr/MIPSPR sensor: (a) adsorption; (b) desorption; and (c) regeneration/equilibrium.

\section{Conclusions}

We successfully constructed a novel SPR sensor for the chiral recognition of L-Trp using a functionalized graphene oxide-MIP composite as a recognition element. The composite was facilely prepared with a new mussel-inspired surface chemistry based on the oxidative polymerization of dopamine. The experimental results indicated that the proposed Gr/MIPSPR sensor exhibited good performance in terms of selectivity and reusability and higher chiral affinity toward L-Trp than toward D-Trp. Our smart chiral sensor would be promising as a novel separator for the direct enantioselective adsorption of amino acids and other chiral compounds.

\section{Conflicts of interest}

There are no conflicts to declare.

\section{Acknowledgements}

This work was supported by the National Natural Science Foundation of China (No. 31501554), Guangdong Provincial Natural Science Foundation of China (2016A030310446), Guangdong Provincial Science and Technology Planning Project of China (2017A020208059).

\section{References}

1 Q. Chen, J. Zhou, Q. Han, Y. Wang and Y. Fu, Colloids Surf., B, 2012, 92, 130-135.

2 L. Guo, Y. Song, H. Yu, L. Pan and C. Cheng, Appl. Surf. Sci., 2017, 407, 82-92.

3 R. Liang, C. Liu, X. Meng, J. Wang and J. Qiu, J. Chromatogr. A, 2012, 1266, 95-102.

4 J. Cubero, F. Toribio, M. Garrido, M. T. Hernandez, J. Maynar, C. Barriga and A. B. Rodriguez, Food Anal. Methods, 2011, 4, 626.

5 Y. Tao, F. Chu, X. Gu, Y. Kong, Y. Lv and L. Deng, Sens. Actuators, B, 2018, 255, 255-261.

6 L. Zhang, C. Xu, C. Liu and B. Li, Anal. Chim. Acta, 2014, 809, 123-127.

7 K. Xu, P. Cheng, J. Zhao and C. Wang, J. Fluoresc., 2011, 21, 991-1000.

8 Y. Wei, A. Tian, Y. Li, X. Wang and B. Cao, J. Mater. Chem., 2012, 22, 8499-8504.

9 G. Yao, R. Liang, C. Huang, Y. Wang and J. Qiu, Anal. Chem., 2013, 85, 11944-11951.

10 P. Englebienne, A. V. Hoonacker and M. Verhas, J. Spectrosc., 2003, 17, 255-273.

11 N. Atar, T. Eren and M. L. Yola, Food Chem., 2015, 184, 7-11.

12 Y. Saylan, S. Akgönüllü, D. Çimen, A. Derazshamshir, N. Bereli, F. Yılmaz and A. Denizli, Sens. Actuators, B, 2017, 241, 446-454.

13 H. E. Indyk and E. L. Filonzi, J. Agric. Food Chem., 2004, 52, 3253-3258.

14 Y. Wang and T. Wei, Chin. Chem. Lett., 2013, 24, 813-816. 
15 M. L. Yola, T. Eren and N. Atar, Sens. Actuators, B, 2015, 210, 149-157.

16 A. M. Shrivastav, S. K. Mishra and B. D. Gupta, Sens. Actuators, B, 2015, 212, 404-410.

17 A. M. Shrivastav, S. K. Mishra and B. D. Gupta, Plasmonics, 2015, 10, 1853-1861.

18 Q. Luo, N. Yu, C. Shi, X. Wang and J. Wu, Talanta, 2016, 161, 797-803.

19 S. Zeng, D. Baillargeat, H. P. Ho and K. T. Yong, Chem. Soc. Rev., 2014, 43, 3426-3452.

20 Z. Zhang, R. Cai, F. Long and J. Wang, Talanta, 2015, 134, 435-442.

21 Y. Yun, M. Pan, G. Fang, Y. Gu, W. Wen, R. Xue and S. Wang, $R S C A d v ., 2018,8,6600-6607$.

22 Y. Liu, L. Zhu, Y. Zhang and H. Tang, Sens. Actuators, B, 2012, 171, 1151-1158.

23 J. Yan, T. Wei, B. Shao, F. Ma, Z. Fan, M. Zhang and F. Wei, Carbon, 2010, 48, 1731-1737.

24 T. Kuila, S. Bose, A. K. Mishra, P. Khanra, N. H. Kim and J. H. Lee, Prog. Mater. Sci., 2012, 57, 1061-1105.

25 L. Xu, W. Yang, K. G. Neoh, E. Kang and G. Fu, Macromolecules, 2010, 43, 8336-8339.

26 J. Chen, R. Liang, X. Wang and J. Qiu, J. Chromatogr. A, 2015, 1409, 268-276.

27 H. Lee, S. M. Dellatore, W. M. Miller and P. B. Messersmith, Science, 2007, 318, 426-430.
28 L. A. Burzio and J. H. Waite, Biochemistry, 2000, 39, 1114711153.

29 M. J. LaVoie, B. L. Ostaszewski, A. Weihofen, M. G. Schlossmacher and D. J. Selkoe, Nat. Med., 2005, 11, 1214.

30 X. Xu, X. Tian, L. Cai, Z. Xu, H. Lei, H. Wang and Y. Sun, Anal. Methods, 2014, 6, 3751-3757.

31 D. C. Marcano, D. V. Kosynkin, J. M. Berlin, A. Sinitskii, Z. Sun, A. Slesarev and J. M. Tour, ACS Nano, 2010, 4, 4806.

32 S. Patra, E. Roy, R. Madhuri and P. K. Sharma, Environ. Sci. Technol., 2015, 49, 6117-6126.

33 S. Patra, E. Roy, R. Madhuri and P. K. Sharma, Anal. Chim. Acta, 2016, 918, 77-88.

34 J. Luo, S. Jiang and X. Liu, J. Phys. Chem. C, 2013, 117, 1844818456.

35 A. C. Ferrari, J. C. Meyer, V. Scardaci, C. Casiraghi, M. Lazzeri and F. Mauri, Phys. Rev. Lett., 2006, 97, 187401.

36 R. Karlsson, A. Michaelsson and L. Mattsson, J. Immunol. Methods, 1991, 145, 229-240.

37 G. K. E. Scriba, Chromatographia, 2012, 75, 815-838.

38 J. Luo, S. Jiang and X. Liu, Sens. Actuators, B, 2014, 203, 782789.

39 L. Li, L. Fan, Y. Dai and X. Kan, Microchim. Acta, 2015, 182, 2477-2483.

40 E. Yılmaz, E. Özgür, N. Bereli, D. Türkmen and A. Denizli, Mater. Sci. Eng., C, 2017, 73, 603. 